\title{
Pathogenic role of endogenous TNF- $a$ in the development of Sjögren's-like sialadenitis and secretory dysfunction in non-obese diabetic mice
}

\author{
Jing Zhou ${ }^{1,2}$, Toshihisa Kawai ${ }^{1,2}$ and Qing $Y u^{1,2}$
}

Patients with Sjögren's syndrome (SS), an autoimmune disease primarily affecting exocrine glands, exhibit enhanced TNF- $a$ expression in the saliva and salivary glands. However, the precise in vivo role of TNF- $a$ during the initiation and development of SS is not clearly defined. The present study is undertaken to determine the function of endogenously produced TNF- $a$ in the pathogenesis of SS in non-obese diabetic (NOD) mice, a model of this human disease. Administration of a neutralizing anti-TNF- $a$ antibody to female NOD mice during the stage prior to disease onset significantly improved salivary secretion, indicating a remission of clinical symptoms of SS. TNF- $a$ blockade also decreased the number of leukocyte foci and reduced the number of T cells and B cells in the submandibular glands (SMG). Moreover, TNF- $a$ blockade reduced T-bet protein levels in the SMG, suggesting a decrease in Thelper 1 and T cytotoxic 1 cells. These cellular changes induced by TNF- $a$ neutralization were associated with a reduction in T- and B-cell chemoattractants CXCL9 and CXC13. In addition, TNF- $a$ blockade markedly increased the expression level of tight junction protein claudin-1 and water channel protein aquaporin-5, two key factors required for normal salivary secretion, in the SMG. Collectively, these findings indicate that endogenous TNF- $a$ has a pathogenic role in the development of SS in the NOD model of this disease.

Laboratory Investigation (2017) 97, 458-467; doi:10.1038/labinvest.2016.141; published online 9 January 2017

Sjögren's syndrome (SS) is a systemic autoimmune disease characterized by inflammation, production of autoantibodies, destruction and dysfunction of exocrine glands, causing xerostomia (dry mouth) and xerophthalmia (dry eyes), as well as systemic symptoms affecting various tissues and organs. ${ }^{1-4}$ Aberrantly activated innate immune cells and autoreactive $\mathrm{T}$ and $\mathrm{B}$ cells have crucial roles in the development of these pathological and functional changes of SS. ${ }^{3,5-9}$ Many cytokines are upregulated in target tissues and circulations of SS patients and a number of them, including IFN- $\alpha$, IFN- $\gamma$, IL-4 and IL-17, are shown to have a critical role in SS pathogenesis in mouse models by affecting the function of the immune cells or exocrine gland tissue cells. $^{7,10-16}$

Tumor necrosis factor- $\alpha$ (TNF- $\alpha$ ) is a pro-inflammatory cytokine that is increased in production in numerous autoimmune or inflammatory diseases. It has a crucial role in the pathogenesis of various autoimmune diseases, such as rheumatoid arthritis, autoimmune psoriasis and Crohn's disease, ${ }^{17-19}$ and anti-TNF- $\alpha$ therapies using monoclonal antibodies or inhibitory molecules have received encouraging results in controlling these diseases. ${ }^{20}$ TNF- $\alpha$ promotes the pathogenesis of these disorders by promoting production of pro-inflammatory cytokines, recruitment of immune cells to local tissues and destruction of organs. ${ }^{18,20}$ In SS patients, elevated TNF- $\alpha$ expression in both salivary glands and serum has been described compared with non-SS sicca patients. ${ }^{21-24}$ TNF- $\alpha$ can be produced by many cell types, including salivary gland epithelium and salivary gland-infiltrating $\mathrm{T}$ helper 1 and $\mathrm{T}$ cytotoxic 1 cells. ${ }^{10,22}$ Multiple in vitro studies have shown that TNF- $\alpha$, alone or cooperating with other inflammatory cytokines, induces apoptosis of human salivary gland cells ${ }^{25}$ and also causes secretory dysfunction in a rat parotid gland Par-C10 cell line by disrupting tight junction integrity and function, which are important for the exchange of water or solutes through paracellular transport. ${ }^{26}$ In addition, TNF- $\alpha$ treatment can augment the production of Ro/SSA and La/SSB in human keratinocytes, two autoantigens

'Department of Immunology and Infectious Diseases, The Forsyth Institute, Cambridge, MA, USA and ${ }^{2}$ Department of Oral Medicine, Infection and Immunity, Harvard School of Dental Medicine, Boston, MA, USA

Correspondence: Dr Q Yu, MD, PhD, Department of Immunology and Infectious Diseases, The Forsyth Institute, 245 First Street, Cambridge, MA 02142, USA.

E-mail: qyu@forsyth.org

Received 25 August 2016; revised 5 November 2016; accepted 20 November 2016 
closely associated with SS disease. ${ }^{27}$ Moreover, in human salivary gland acinar cells, TNF- $\alpha$ treatment downregulates the expression of aquaporin 5 (AQP5), a water channel protein required for salivary secretion. ${ }^{28-31}$ Taken together, these lines of evidence suggest that TNF- $\alpha$ may be a pathogenic factor in SS and its inhibition may impede the development or attenuate the severity of this disease.

Both laboratory and clinical studies have suggested a possible therapeutic effect of anti-TNF- $\alpha$ treatment in SS. A study using a SS mouse model demonstrated that inhibition of TNF- $\alpha$ with pegylated human TNF receptor 1 restrains the development of SS, as demonstrated by reduced lymphocyte infiltration and decreased expression of inflammatory cytokines in lacrimal and salivary glands. ${ }^{32,33}$ A monoclonal antiTNF- $\alpha$ antibody attenuated the sicca symptoms in primary SS patients in a small and open-label pilot clinical study. ${ }^{32}$ However, the same antibody did not show a clear efficacy in another randomized and double-blind controlled trial in primary SS patients. ${ }^{33}$ The specific in vivo role of TNF- $\alpha$ in the pathogenesis of SS, especially in the stage of development prior to the disease onset, has not been clearly defined.

In the present study, we investigated the role of endogenous TNF- $\alpha$ in the development of SS using a neutralizing anti-TNF- $\alpha$ antibody in non-obese diabetic (NOD) mice, a model of SS-like disease. We demonstrated that endogenous TNF- $\alpha$ serves as an important pathogenic mediator in the development of this disease.

\section{MATERIALS AND METHODS}

Mice

Female NOD mice, strain NOD/ShiLtJ, were purchased from the Jackson Laboratory and kept under specific pathogen-free conditions. All the experimental protocols were approved by the Institutional Animal Care and Use Committee of the Forsyth Institute. All the procedures were performed in compliance with the National Institutes of Health guidelines for the care and use of laboratory animals.

\section{Antibodies}

Purified monoclonal anti-mouse TNF- $\alpha$ (TN3-19.12) and its isotype control hamster IgG used for injection were purchased from BioXCell. For flow cytometry, anti-CD4 and anti-CD8 antibodies were purchased from BioLegend. For immunohistological chemistry, biotin-conjugated antiCD4 antibody and anti-CD8 antibody were obtained from eBioscience, anti-T-bet- and biotin-conjugated anti-B220 antibodies were from BioLegend, and biotin-conjugated anti-hamster IgG was from Vector Laboratories. For immunofluorescence staining, anti-AQP5 and Alexa Fluor647conjugated rabbit IgG were purchased from Abcam.

\section{In vivo Administration of Anti-TNF-a Antibody}

In all, $200 \mu \mathrm{g}$ anti-mouse TNF- $\alpha$ antibody or control hamster IgG were intraperitoneally (i.p.) administered to 4-week-old female NOD mice 3 times weekly for 6 weeks. All the analyses were performed 2 days after the last injection.

\section{Histological, Immunohistochemical (IHC) and Immunofluorescense Staining}

Harvested SMG tissues were fixed in 4\% paraformaldehyde, embedded in paraffin and sectioned to $5 \mu \mathrm{m}$ thickness. The sections were subsequently stained with hematoxylin and eosin (H\&E) and assessed for leukocyte infiltration. Leukocyte focus is defined as leukocyte aggregate that contains at least 50 leukocytes. The numbers of leukocyte foci in each of the three non-consecutive sections of each SMG sample were counted, and the highest number among the three was used for further calculation of leukocyte focus score, which is defined as the number of leukocyte foci per $4 \mathrm{~mm}^{2}$ area of the tissue section. For IHC staining, the sections were de-paraffinized and stained with antibodies against mouse CD4, CD8, T-bet or B220 at $4{ }^{\circ} \mathrm{C}$ overnight using the VECTASTAIN Elite ABC Kit (Vector Laboratories) following the manufacturer's manual. For immunofluorescense staining, the deparaffinized sections were incubated with anti-AQP5 antibody (Abcam) after antigen retrieval. They were then incubated with Alexa Fluor 647-conjugated mouse IgG (Abcam) and imaged using a Leica laser scanning confocal microscope (Zeiss). Quantification of the positively stained areas in the IHC-stained tissue sections was performed using the ImageJ 1.50i software. Briefly, images of IHC were saved as RGB Tiff files and the brown-stained areas were segmented using appropriate color thresholding. The percentage of thresholded area in each image was measured and calculated. Images from immunofluorescence-stained samples were converted into grayscale (8-bit) and the gray color was segmented using thresholding. Integrated density measurement was performed to determine the fluorescence intensity of the staining.

\section{Detection of Serum Antinuclear Antibodies (ANA)}

Serum ANA was assessed using HEp-2 human epithelial cell substrate slides (INOVA Diagnostics), according to the manufacturer's instructions. After staining, the images were acquired on an inverted wide-field fluorescence microscope (Zeiss) at $\times 400$ magnification and processed using the Zeiss software (ZEN blue edition).

\section{ELISA}

The M3 muscarinic acetylcholine receptor (M3R) peptide solution $(2 \mu \mathrm{g} / \mathrm{ml})$ was adsorbed onto a Nunc MaxiSorp flatbottom 96-well plate (BioLegend). None-specific binding sites on the plate were blocked with ELISA Assay Diluent buffer (BioLegend). Sera (1:6 diluted) were added to the plate and incubated over night at $4{ }^{\circ} \mathrm{C}$ After washing with $0.05 \%$ Tween-20 in PBS, the plate was incubated with 1:300 diluted biotinylated goat anti-mouse IgG antibody (Vector Labratories) for $1 \mathrm{~h}$. After washing, the plate was incubated with Avidin-HRP solution for $30 \mathrm{~min}$, and the bound 

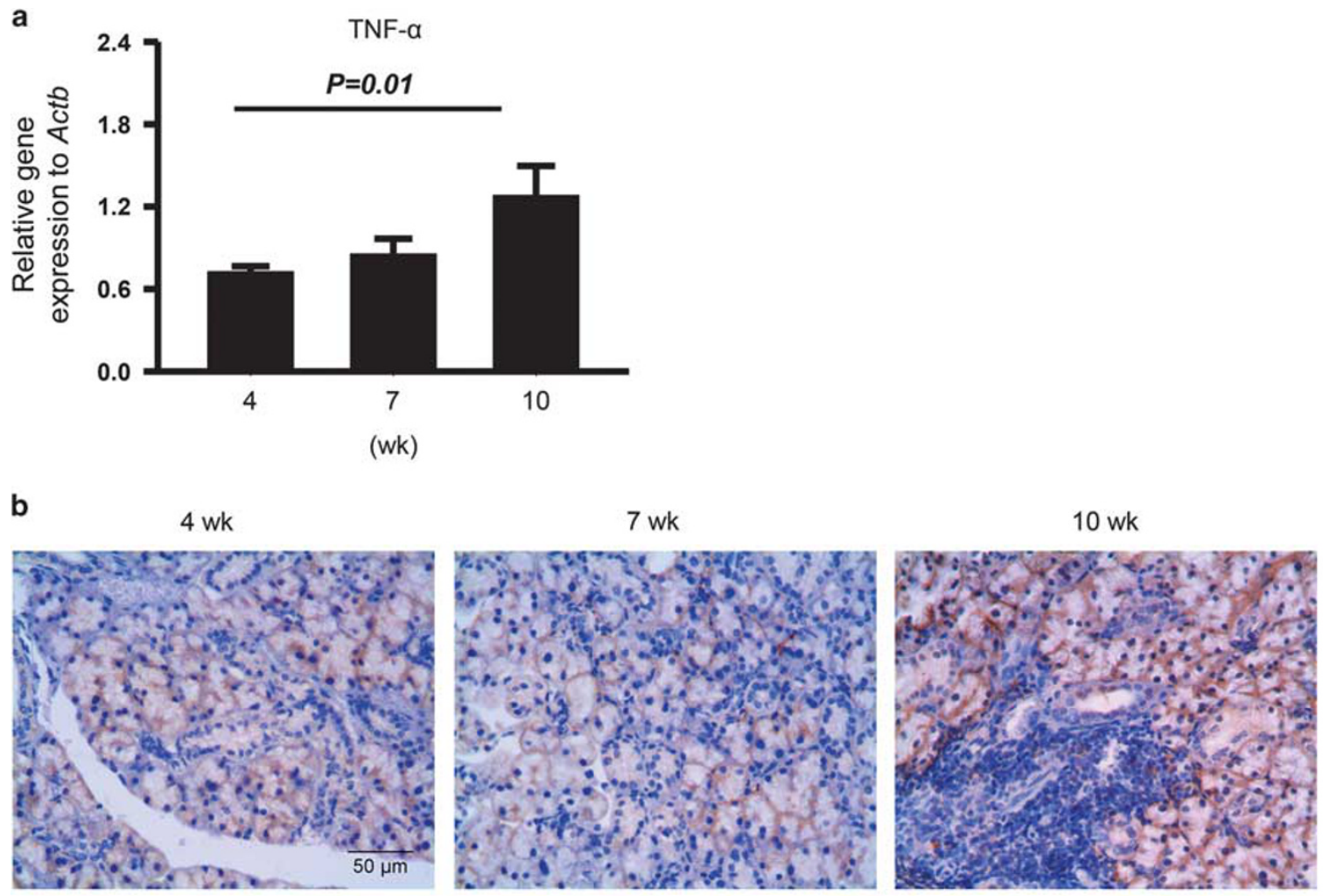

TNF- $\alpha$ IHC
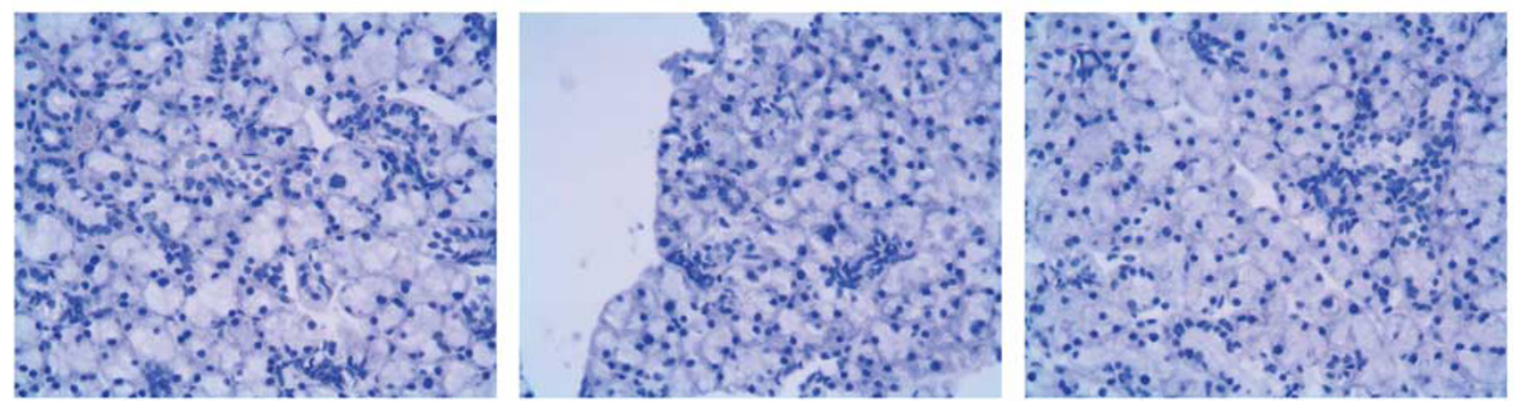

IHC control

Figure 1 Expression of TNF- $a$ in the submandibular glands (SMG) of NOD mice. (a) Real-time PCR analysis of TNF- $a$ mRNA level in the SMG of NOD mice aged 4, 7 and 10 weeks. The expression level was presented relative to that of $\beta$-actin. Error bars represent the s.e.m. (b) IHC staining of TNF- $a$ protein in SMG sections of NOD mice aged 4, 7 and 10 weeks. Original magnification: $\times 400$. Data are the average of analyses of 4-7 mice for each treatment group.

antibodies on plate detected by the TMB substrate. Finally, the reaction was stopped by adding $100 \mu \mathrm{l} 2 \mathrm{~N} \mathrm{H}_{2} \mathrm{SO}_{4}$ to each well and the absorbance at $450 \mathrm{~nm}$ was read with a microplate reader (BioTek).

\section{Measurement of Salivary Flow Rate}

TNF- $\alpha$-treated NOD mice were weighed and given an i.p. injection of $100 \mu \mathrm{l}$ PBS-based secretagogue solution containing isoproterenol $(0.02 \mathrm{mg} / \mathrm{ml})$ and pilocarpine $(0.05 \mathrm{mg} / \mathrm{ml})$. Saliva was collected continuously for $5 \mathrm{~min}$ from the oral cavity of mice with a micropipette, starting from 1 min after secretagogue injection. The volume of saliva was measured and normalized to the body weight.

\section{Real-time RT-PCR}

Total RNA was isolated using the RNA Kit (Qiagen) and reverse transcribed into CDNA by MLV reverse transcriptase (Promega). SYBR Green-based real-time PCR amplification (Qiagen) was performed for 40 cycles with annealing and extension temperature at $60^{\circ} \mathrm{C}$, on a LightCycler 480 RealTime PCR System (Roche). Primer sequences are: mouse CXCL9, forward, 5'-CCCTCAAAGACCTCAAACAGT-3'; reverse, 5'-AGTCCGGATCTAGGCAGGTT-3'; CXCL10 forward, $5^{\prime}$-CCAGTGAGAATGAGGGCCAT-3', reverse, $5^{\prime}$-CCG GATTCAGACATCTCTGC-3'; CXCL11 forward, 5'-GCAGA GATCGAGAAAGCTTCT-3', reverse, 5'-GTCCAGGCACCTT TGTCGTT-3'; and CXCL13, forward, 5'-AGATCGGATTC 
AAGTTACGCC-3' , reverse, $5^{\prime}$-TTTGGCACGAGGATTCACA CA.-3'. All transcript levels were normalized to $\beta$-actin.

\section{Statistical Analysis}

All statistical significance was determined by Student's $t$-test (two-tailed, two-sample equal variance). $P$-values $<0.05$ were considered as statistically significant.

\section{RESULTS}

Expression of TNF- $a$ in the Submandibular Glands (SMG) of NOD Mice Increases with Age

Expression of TNF- $\alpha$ is elevated in saliva and salivary glands of SS patients. To assess whether TNF- $\alpha$ is increased in the SMG during the development of SS disease in NOD mice, we examined TNF- $\alpha$ expression levels in the SMG of NOD mice at 4, 7 and 10 weeks of age. Our characterization of the kinetics of the disease development in female NOD mice shows that, at 10 weeks of age, the majority of the mice have leukocyte infiltrates in the SMG, easily detectable ANA in the serum and reduced salivary flow rate compared with control Balb/c mice (data not shown). Hence, the female NOD mice used in this study have the onset of the disease around 10 weeks of age. Real-time PCR analysis and IHC staining showed that both the mRNA amount and the protein level of TNF- $\alpha$ in the SMG increased significantly over time between 4 and 10 weeks of age (Figures $1 \mathrm{a}$ and $\mathrm{b}$ ). TNF- $\alpha$ was mainly expressed by antigen-presenting cells that interspersed among the epithelial cells (Figure 1b). At 10 weeks of age, SMGinfiltrating leukocytes were present in most of the mice and some of them also expressed TNF- $\alpha$ (Figure 1b). Hence, TNF- $\alpha$ expression increases with the spontaneous development of SS-like exocrinopathy in NOD mice, suggesting a possible role of TNF- $\alpha$ in the pathogenesis of this disease.

\section{Blockade of Endogenous TNF-a Reduces Leukocyte Infiltration of SMG and Improves Salivary Secretion in NOD Mice}

To determine whether endogenous TNF- $\alpha$ is important for the pathogenesis of SS, we neutralized TNF- $\alpha$ activity by i.p. administration of an anti-TNF- $\alpha$ antibody or its isotype control hamster IgG into 4 week-old female NOD mice, 3 times weekly for 6 weeks, and analyzed the characteristic pathologies of SS. To determine whether systemically injected anti-TNF- $\alpha$ antibody can directly target SMG tissues, the presence of hamster IgG in this gland was examined by IHC staining. The results showed detectable hamster IgG in the SMG of both anti-TNF- $\alpha$ - and control hamster IgG-treated mice, indicating that the injected antibody was delivered into target SMG as intended (Figure 2a). We then examined the effect of TNF- $\alpha$ neutralization on the leukocyte infiltration of SMG, one of the characteristic pathologies of SS. Histological analysis showed that blockade of TNF- $\alpha$ significantly reduced the leukocyte focus score, defined as the number of leukocyte foci per $4 \mathrm{~mm}^{2}$ area of the SMG tissue section (Figure 2b). Impaired salivary secretion is one of the major disease manifestations of SS, and we found that antiTNF- $\alpha$ treatment markedly improved the stimulated salivary flow rate (Figure 2c). Therefore, blockade of endogenous TNF- $\alpha$ impedes the development and onset of SS-like sialadenitis and hyposalivation in NOD mice.

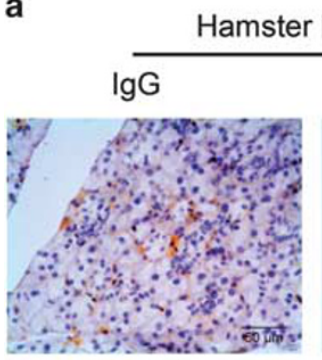

b

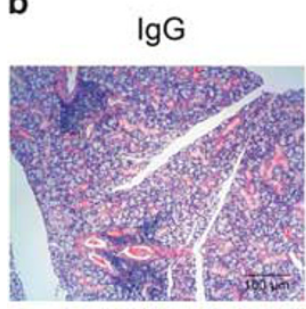

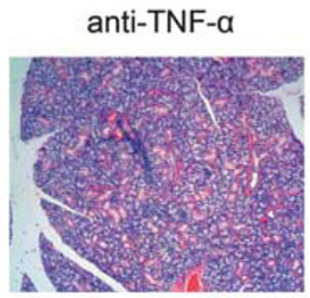

anti-TNF-a

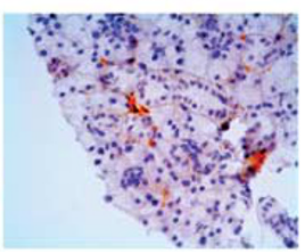

anti-TNF- $\alpha$

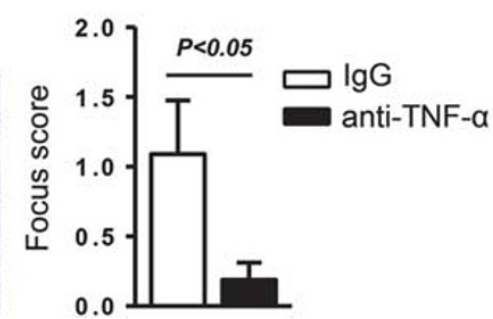

IHC control
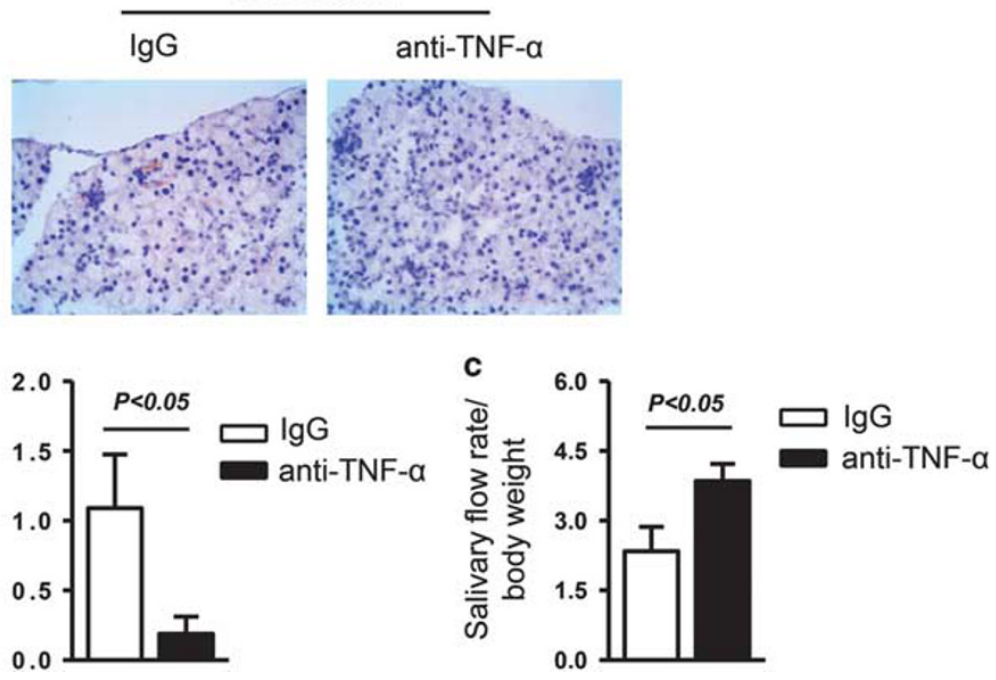

Figure 2 Blockade of TNF- $a$ impedes the development of SS-like pathologies. Anti-TNF- $a$ or control lgG was injected into 4-week-old NOD mice 3 times weekly for 6 weeks. (a) The presence of the injected antibody and control lgG in the SMG was examined by IHC staining with an anti-hamster lgG antibody. Original magnification: $\times 400$. (b) Images of H\&E staining of SMG sections with original magnification $\times 200$. Bar graph shows the mean leukocyte focus score. (c) Stimulated saliva flow rate normalized to body weight. All data in this figure are representative or the average of analyses of 6-13 mice for each treatment group. Error bars represent the s.e.m. 
a

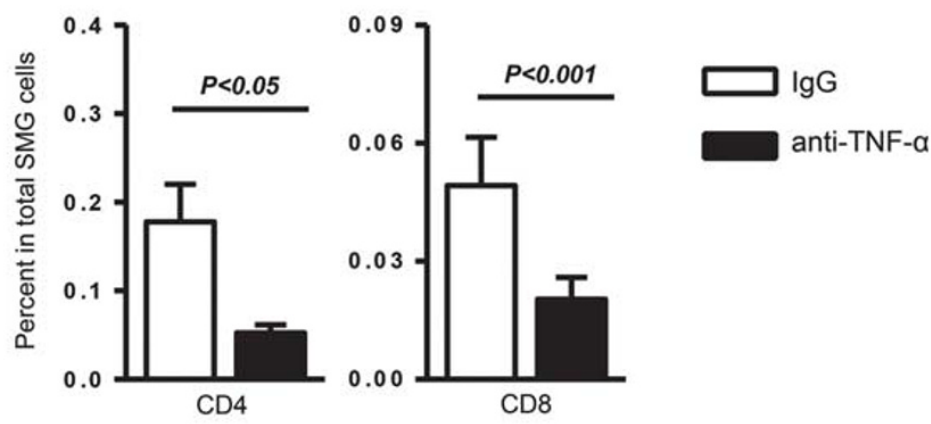

b

$\lg G$
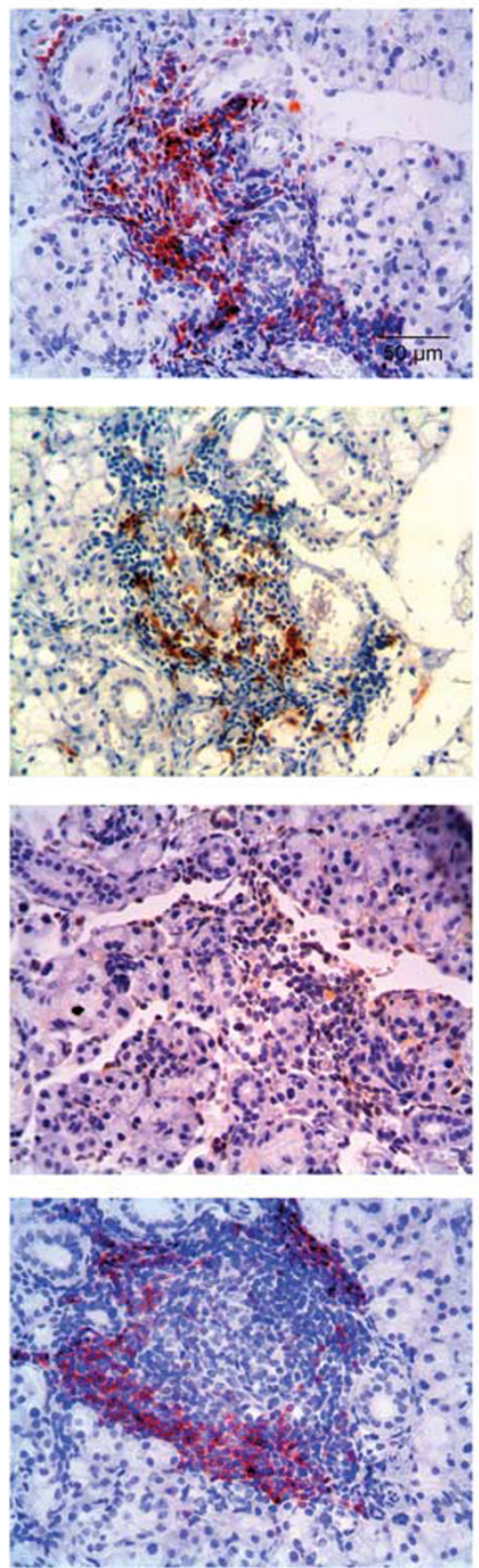

anti-TNF- $\alpha$

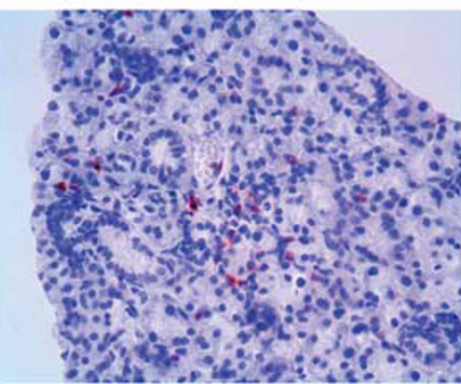

CD4

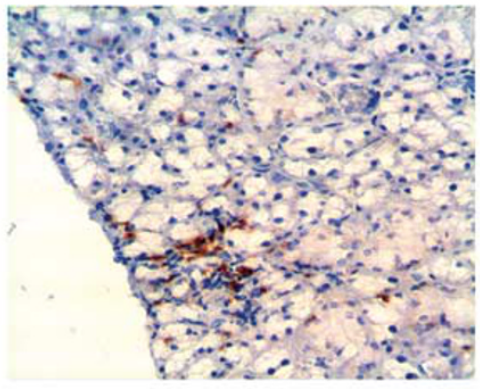

CD8

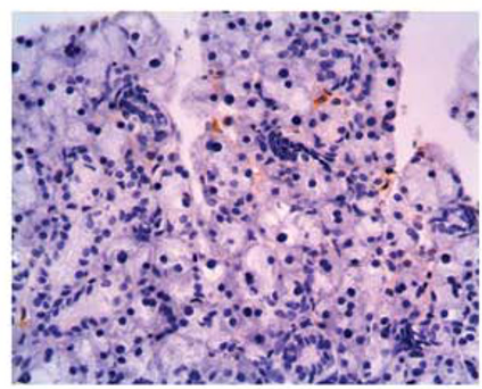

T-bet

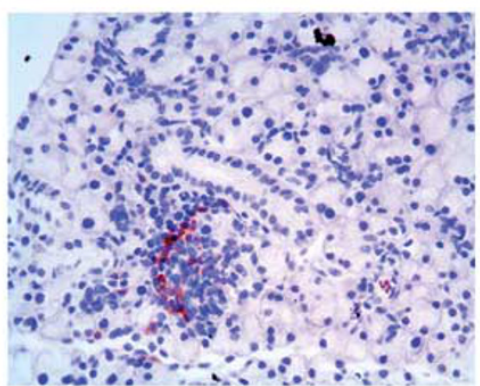

B220

Figure 3 Neutralization of TNF- $a$ decreases the number of SMG-infiltrating T and B cells in the SMG. Anti-TNF- $a$ or control lgG was injected into 4week-old NOD mice 3 times weekly for 6 weeks. (a) Flow cytometric analysis of the percentage of CD4 and CD8 T cells among total SMG cells. (b) IHC staining of CD4, CD8, T-bet and B cells in SMG sections. Original magnification: $\times 400$. All data in this figure are representative or the average of analyses of 6 mice for each treatment group. 


\section{Blockade of TNF-a Causes a Reduction in SMG- Infiltrating $T$ and $B$ Cells}

We next assessed the effect of TNF- $\alpha$ blockade on lymphocyte subsets in the SMG. Flow cytometric analysis revealed that the percentage of CD4 and CD8 T cells among total SMG cells was significantly decreased by anti-TNF- $\alpha$ treatment (Figure 3a). Consistent with these results, IHC staining of SMG sections detected fewer CD4- or CD8-positive cells in the SMG of anti-TNF- $\alpha$ mice compared with the IgG-treated control mice (Figure 3b). Moreover, TNF- $\alpha$ blockade reduced the number of T-bet-expressing cells in the SMG, suggesting an overall decrease in T helper 1- and/or T cytotoxic 1 cells (Figure $3 \mathrm{~b}$ ). In addition, IHC staining results also provided evidence that anti-TNF- $\alpha$ antibody led to a decreased number of $\mathrm{B}$ cells in the SMG, defined as $\mathrm{B}_{220^{+}}$cells (Figure $3 \mathrm{~b}$ ). Hence, the protective function of TNF- $\alpha$ blockade in SS development is associated with reduced amount of SMGinfiltrating $\mathrm{T}$ and $\mathrm{B}$ cells.

\section{TNF-a Blockade Leads to a Reduced Expression of T- and B-cell Chemoattractants in the SMG}

Having shown that TNF- $\alpha$ blockade decreased the number of $\mathrm{T}$ and $\mathrm{B}$ cells in the SMG, we further assessed whether this is due to a reduced recruitment of these cells resulting from downregulation of T- and B-cell chemoattractants. Real-time PCR analysis showed that TNF- $\alpha$ blockade significantly reduced the mRNA level of CXCL9 while having no effect on that of CXCL10 or CXCL11, in the SMG (Figure 4), suggesting that T-cell chemoattractant CXCL9 but not CXCL10 or CXCL11 was involved in the inhibition of T-cell trafficking into SMG as a result of TNF- $\alpha$ blockade. Furthermore, consistent with the reduction in B-cell numbers in SMG, mRNA level of B-cell chemoattractant CXCL13 was significantly decreased as a result of TNF- $\alpha$ blockade (Figure 4). Collectively, these results suggest that the antiinflammatory role of TNF- $\alpha$ blockade may be mediated, at least in part, by downregulating CXCL9 and CXCL13 expression in the SMG.

\section{Blockade of TNF-a Increases Claudin-1 and AQP5 levels in the SMG}

Disruption of tight junction integrity has been reported in the salivary glands of SS patients ${ }^{34}$ and is associated with impaired secretory function in rat salivary gland cells. ${ }^{26}$ To determine whether TNF- $\alpha$ blockade affects the tight junction integrity in the SMG, we performed IHC staining for claudin-1, claudin-2 and occludin in SMG tissue sections. TNF- $\alpha$ blockade led to a markedly increased level of claudin-1 (Figure 5a), whereas it did not alter that of claudin-2 or occludin (data not shown). Hence, the improvement of salivary secretion upon TNF- $\alpha$ blockade is associated with increased claudin-1 expression in the SMG.

We next examined the expression of AQP5, a water channel protein that is critical for the normal salivary secretion. ${ }^{28-30}$ Immunofluorescence staining of SMG sections showed a higher level of AQP5 in anti-TNF- $\alpha$-treated mice than in IgG-treated controls (Figure 5b). Thus elevated AQP5 expression could be another mechanism of anti-TNF- $\alpha$ induced improvement of salivary secretion.

\section{Blockade of TNF-a Leads to Enhanced Autoantibody Production}

We next assess the effect of TNF- $\alpha$ neutralization on serum autoantibody levels, a characteristic parameter of SS. Using HEp-2 cells as substrates, our analysis showed that sera from anti-TNF- $\alpha$-treated mice exhibited higher levels of ANA to different extents than those from IgG-treated mice (Figure 6a). Moreover, autoantibodies against M3R, which can contribute to salivary gland hypofunction, ${ }^{35-37}$ were also increased by TNF- $\alpha$ blockade (Figure 6b). These results are consistent with previous reports that anti-TNF treatment leads to increased autoantibody production in a number of other autoimmune disorders, ${ }^{38}$ indicating complex and multifaceted effects of TNF- $\alpha$ in these disease conditions.

\section{DISCUSSION}

This study demonstrates a crucial pathogenic role of endogenous TNF- $\alpha$ in the development and onset of SS-like exocrinopathy and secretory dysfunction in NOD mice and

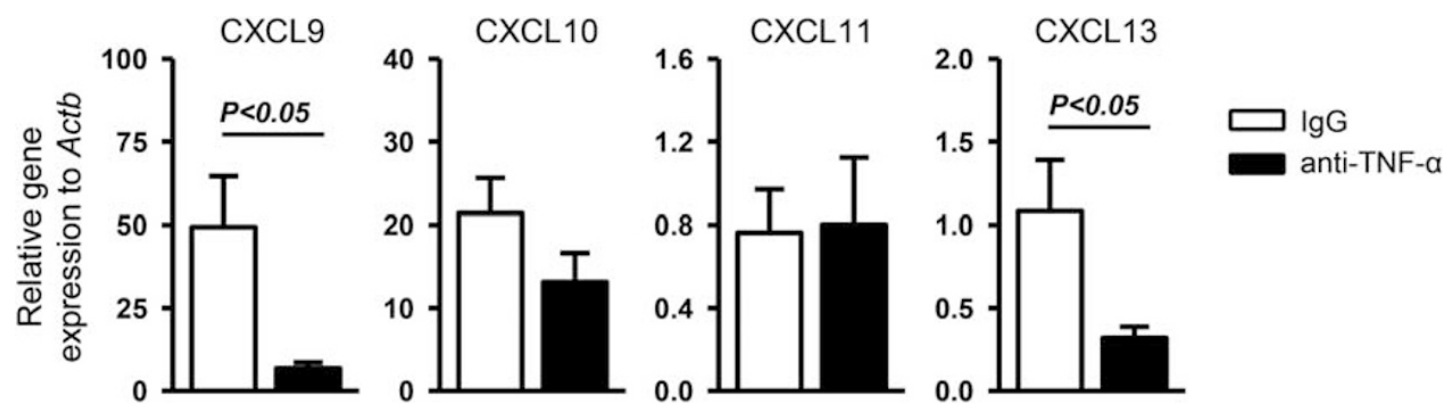

Figure 4 TNF- $a$ blockade reduces the expression of T- and B-cell chemoattractants in the SMG. Anti-TNF- $a$ or control lgG was injected into 4-week-old NOD mice 3 times weekly for 6 weeks. Real-time PCR analysis of T- and B-cell-associated chemoattractant expressions in SMG, presented relative to that of $\beta$-actin. Data are the average of analyses of 13-15 mice for each group. 
a
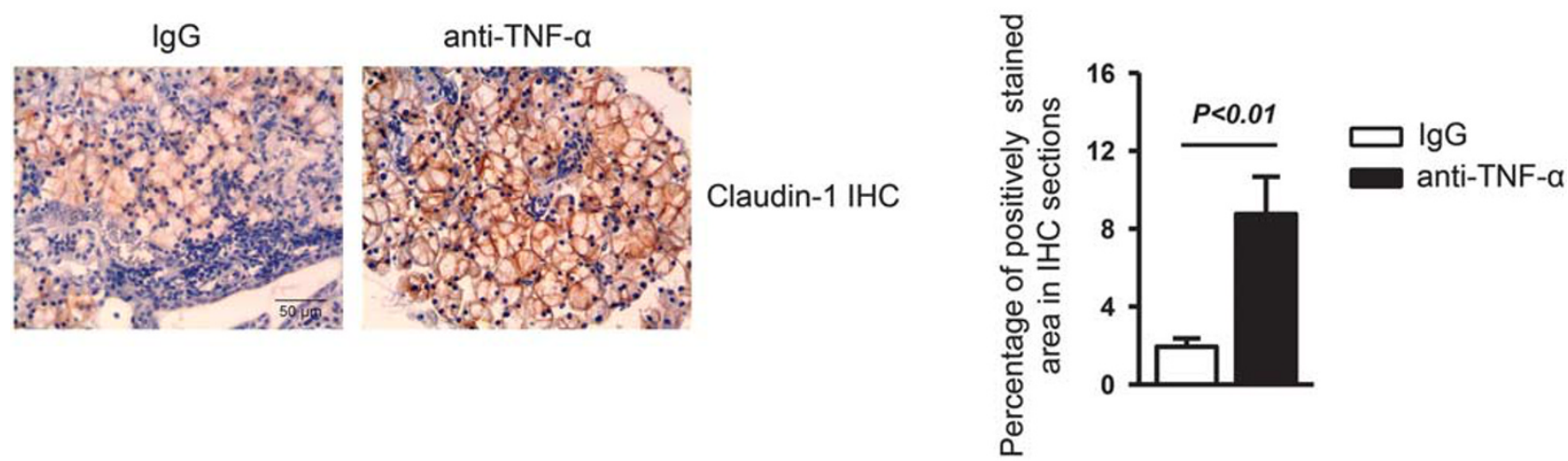

b
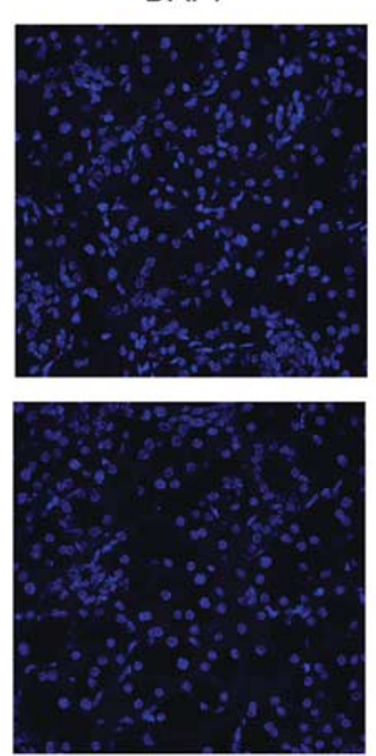

AQP5
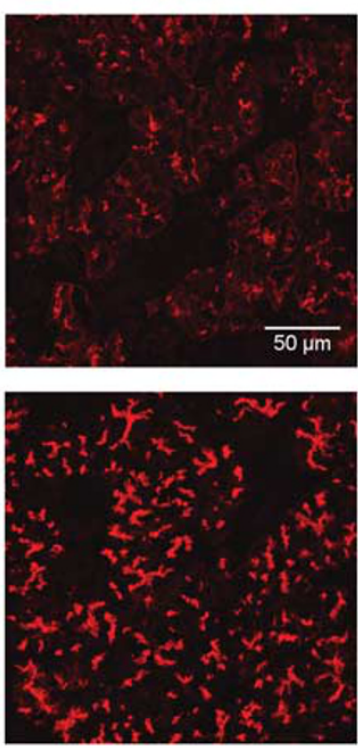
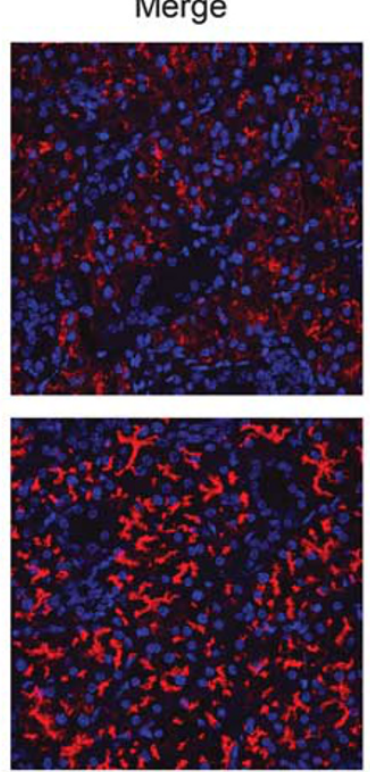

$\lg G$

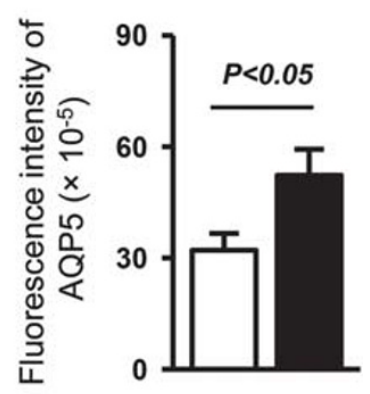

anti-TNF- $\alpha$

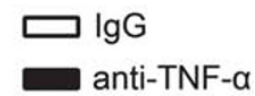

Figure 5 Neutralization of TNF- $a$ increases claudin-1 and AQP5 protein levels in the SMG. Anti-TNF- $a$ or control lgG was injected into 4-week-old NOD mice 3 times weekly for 6 weeks. (a) IHC of claudin-1 protein in SMG sections. Original magnification: $\times 400$. Bar graph shows the percentage of positively stained areas in the IHC-stained sections. (b) Immunofluoresence staining of AQP5 protein in SMG sections. Original magnification: $\times 400$. Bar graph shows the fluorescence intensity of AQP5 staining. All data in this figure are representative or the average of analyses of 13-15 mice for each group.

identifies several potential underlying mechanisms of TNF- $\alpha$ function, including downregulation of $\mathrm{T}$ - and $\mathrm{B}$-cell chemoattractants and upregulation of tight junction protein claudin-1 and water channel protein AQP5.

Previous studies have shown an elevation in TNF- $\alpha$ levels in the salivary glands and sera of SS patients. ${ }^{21-24}$ We further showed in this study that salivary gland TNF- $\alpha$ expression increases with age in NOD mice accompanying SS development, supporting a possible pathogenic role of this cytokine in SS, especially in the stage prior to the disease onset at 10 weeks of age. By inhibiting TNF- $\alpha$ function between 4 and 10 weeks of age in NOD mice, we showed that it is required for the full development of SS-like inflammation and secretory dysfunction of salivary glands. A previous report has shown that pegylated human soluble TNF receptor 1 (TNFR1), which prevents the binding of TNF- $\alpha$ to TNFR1, inhibits inflammation and secretory dysfunction of exocrine glands in NOD mice. ${ }^{39}$ As TNF- $\alpha$ can also bind to TNFR2, our study is important in elucidating the overall function of TNF- $\alpha$, which engages both TNFR1 and TNFR2, in the development and onset of SS. Another study has examined the effect of local, salivary gland-specific expression of a TNF receptor 1:IgG fusion protein (TNFR1:IgG), which inhibits TNF- $\alpha$ activity, on SS in NOD mice and showed a complex, varying effect on the salivary secretion depending on the age window and length of local TNFR1:IgG expression. ${ }^{40}$ At early ages, a short period of TNFR1:IgG expression appears to improve salivary secretion, consistent with our finding that 


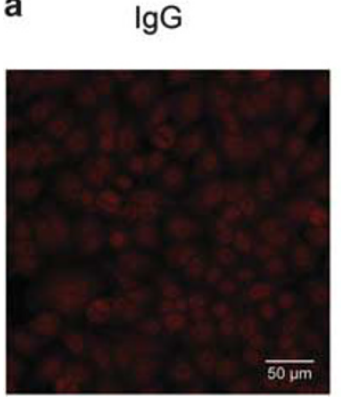

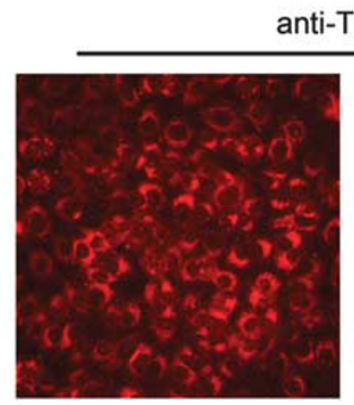

anti-TNF- $\alpha$

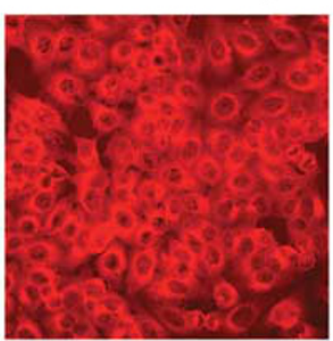

b

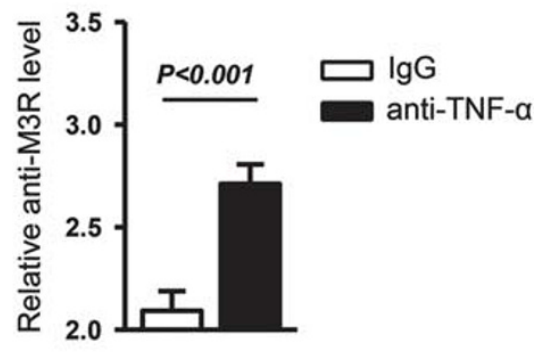

Figure 6 Blockade of TNF-a promotes autoantibody production. Anti-TNF- $a$ or control lgG was injected into 4-week-old NOD mice 3 times weekly for 6 weeks. (a) Detection of serum ANA. Original magnification: $\times 400$. (b) Serum anti-M3R autoantibody level was determined by ELISA. All data in this figure are representative or the average of analyses of 13-15 mice for each group.

also examined the effect of TNF- $\alpha$ at the early stage prior to the disease onset. ${ }^{40}$ The same report shows that local TNFR1: IgG expression does not affect tissue inflammation and autoantibody production, unlike what our study shows. This difference is likely attributed to the different approaches and timing of TNF- $\alpha$ blockade employed. TNF- $\alpha$ can be produced by and affect many cell types at various sites, and thus systemic blockade of TNF- $\alpha$ we employed is likely inhibiting a wider range of TNF- $\alpha$ actions than the local inhibition approach. Moreover, our study specifically blocked TNF- $\alpha$ at an earlier stage prior to SS onset, whereas the other study blocked this cytokine at a later stage for a much longer period of time. The effects of anti-TNF- $\alpha$ antibody we used in this study at later stages of the SS disease will be tested in our future studies by using NOD-derived strains that develop SS but not diabetes.

It is important to note that our study excluded the interference from the clinical type-1 diabetes, which the majority of female NOD mice exhibit after 12-16 weeks of age, later than the onset of SS and our end point analysis. Therefore, the inhibitory effect of anti-TNF- $\alpha$ antibody in the development and onset of SS disease is not an indirect consequence of its effect on type-1 diabetes.

In characterizing the potential mechanisms by which TNF- $\alpha$ blockade reduces SMG inflammation, we found that local expression of CXCL9 and CXCL13, two key chemokines that can attract T and B cells, is downregulated. TNF- $\alpha$ can upregulate CXCR3 ligand expression to facilitate leukocyte infiltration of target organs in various autoimmune or inflammatory disease conditions. ${ }^{41,42}$ Interestingly, other reports have shown that TNF- $\alpha$ is not a potent inducer of CXCL9 expression, ${ }^{43}$ whereas we showed that, in the SS disease setting, TNF- $\alpha$ has an indispensable role in the optimal expression of CXCL9, but not CXCL10 and CXCL11. These findings collectively point to a disease contextdependent function of TNF- $\alpha$ in promoting the expression of these chemokines. Whether the induction of CXCL9 by TNF- $\alpha$ requires cooperation of other factors, such as IFN- $\gamma$, during the development of SS will be addressed in our future investigations. In addition to CXCR3 ligands, CXCL13 is a critical chemokine directing B-cell recruitment to target organs in inflammatory conditions ${ }^{44}$ and the decrease in CXCL13 expression resulting from anti-TNF therapy is considered a potential predictive biomarker in rheumatic arthritis patients. ${ }^{45}$ In line with these findings, we demonstrated that neutralization of TNF- $\alpha$ leads to downregulation of CXCL13 expression also in SS disease settings. The cellular and molecular basis of TNF- $\alpha$ induction of CXCL9 and CXCL13 will be a subject of our future investigations.

Tight junctions are protein complexes that form a wellregulated diffusion barrier between the apical and basal regions of exocrine gland cells and are crucial for water and solute exchange. ${ }^{46}$ In vitro studies have shown that TNF- $\alpha$ impairs the secretory function in rat salivary gland epithelial cells, which is associated with the disruption of tight junction. ${ }^{26,46}$ Here we provided in vivo evidence that the improvement in salivary secretion resulting from TNF- $\alpha$ blockade is accompanied by elevated expression of tight junction protein claudin-1, which has a critical role in maintaining tight junction integrity. ${ }^{46}$ Collectively, these findings suggest that TNF- $\alpha$ promotes the disruption of barrier function in salivary gland tissues by downregulating claudin-1 expression, which in turn contributes to defective salivary secretion. Meanwhile, we also observed that TNF- $\alpha$ neutralization increases SMG expression of AQP5, a water channel protein vital for normal salivary secretion. ${ }^{28-30}$ AQP5 deficiency leads to impaired salivary secretion owing to defective water export in salivary gland epithelial cells, ${ }^{29,30}$ which can be corrected by local gene therapy that overexpresses AQP $1 .{ }^{28}$ Therefore, our finding suggests that TNF- $\alpha$ promotes the secretory dysfunction of salivary glands, at least in part, by downregulating AQP5 expression.

Anti-TNF- $\alpha$-based therapy has been successfully used in treating rheumatic arthritis, inflammatory bowel disease and systemic lupus erythematosus. An unintended and poorly understood consequence of this treatment is the development of lupus-like changes, termed as anti-TNF- $\alpha$-induced lupus erythematosus, in a small proportion of patients. ${ }^{38,47}$ These patients transiently develop ANA and anti-double-stranded DNA antibodies, which fall back to baseline levels shortly 
after the cessation of anti-TNF- $\alpha$ treatment, generally without clinical manifestations of lupus. ${ }^{47-49}$ Consistent with these reports, here we show that, while TNF- $\alpha$ blockade mitigates inflammation and dysfunction of salivary glands, it causes a marked increase in serum ANA and anti-M3R autoantibody levels. The functional significance and the underlying mechanisms of the excessive autoantibody production induced by anti-TNF- $\alpha$ treatment in the SS setting are currently unclear and require further determination.

To date, a few clinical trials on anti-TNF- $\alpha$ therapies in SS disease have been conducted and generated inconsistent results on the efficacy. ${ }^{32,33}$ In order to develop anti-TNF- $\alpha$ therapy with better and reliable efficacy, it will be important to assess the effect of anti-TNF- $\alpha$ in mouse models with established SS disease to gain better understanding of its action at this stage and to assess the efficacy of the combination of anti-TNF- $\alpha$ with other immunemanipulating strategies in ameliorating this disease.

\section{Conclusions}

This study demonstrates that neutralization of TNF- $\alpha$ during the stage prior to disease onset impedes the development and onset of SS-like salivary gland inflammation and secretory dysfunction in NOD mice, indicating that endogenous TNF- $\alpha$ has a critical pathogenic role in the development and onset of this disease.

\section{ACKNOWLEDGMENTS}

We thank Juan Du and Dr Atsushi Ikeda for technical assistance in this work. This study was supported by the grant from the National Institutes of Health to QY (R01 DE023838).

\section{DISCLOSURE/CONFLICT OF INTEREST}

The authors declare no conflict of interest.

1. Lee BH, Tudares MA, Nguyen CQ. Sjogren's syndrome: an old tale with a new twist. Arch Immunol Ther Exp (Warsz) 2009;57:57-66.

2. Fox PC. Autoimmune diseases and Sjogren's syndrome: an autoimmune exocrinopathy. Ann NY Acad Sci 2007;1098:15-21.

3. Voulgarelis $M$, Tzioufas AG. Pathogenetic mechanisms in the initiation and perpetuation of Sjogren's syndrome. Nat Rev Rheumatol 2010;6: 529-537.

4. Theander E, Manthorpe R, Jacobsson LT. Mortality and causes of death in primary Sjogren's syndrome: a prospective cohort study. Arthritis Rheum 2004;50:1262-1269.

5. Singh N, Cohen PL. The T cell in Sjogren's syndrome: force majeure, not spectateur. J Autoimmun 2012;39:229-233.

6. Li H, Dai M, Zhuang Y. A T cell intrinsic role of Id 3 in a mouse model for primary Sjogren's syndrome. Immunity 2004;21:551-560.

7. Youinou P, Pers JO. Disturbance of cytokine networks in Sjogren's syndrome. Arthritis Res Ther 2011;13:227.

8. Katsifis GE, Moutsopoulos NM, Wahl SM. T lymphocytes in Sjogren's syndrome: contributors to and regulators of pathophysiology. Clin Rev Allergy Immunol 2007;32:252-264.

9. Hayakawa I, Tedder TF, Zhuang Y. B-lymphocyte depletion ameliorates Sjogren's syndrome in Id3 knockout mice. Immunology 2007;122: 73-79.

10. Jin JO, Yu Q. T cell-associated cytokines in the pathogenesis of Sjogren's syndrome. J Clin Cell Immunol 2013;S1:11742.

11. Szczerba BM, Rybakowska PD, Dey P, et al. Type I interferon receptor deficiency prevents murine Sjogren's syndrome. J Dent Res 2013;92: 444-449.
12. Roescher N, Tak PP, Illei GG. Cytokines in Sjogren's syndrome: potential therapeutic targets. Ann Rheum Dis 2010;69:945-948.

13. Brayer JB, Cha S, Nagashima $\mathrm{H}$, et al. IL-4-dependent effector phase in autoimmune exocrinopathy as defined by the NOD.IL-4-gene knockout mouse model of Sjogren's syndrome. Scand J Immunol 2001;54: 133-140.

14. Cha S, Brayer J, Gao J, et al. A dual role for interferon-gamma in the pathogenesis of Sjogren's syndrome-like autoimmune exocrinopathy in the nonobese diabetic mouse. Scand J Immunol 2004;60:552-565.

15. Nguyen $\mathrm{CQ}$, Yin $\mathrm{H}$, Lee $\mathrm{BH}$, et al. Pathogenic effect of interleukin-17A in induction of Sjogren's syndrome-like disease using adenovirusmediated gene transfer. Arthritis Res Ther 2010;12:R220.

16. Nguyen CQ, Yin H, Lee BH, et al. IL17: potential therapeutic target in Sjogren's syndrome using adenovirus-mediated gene transfer. Lab Invest 2011;91:54-62.

17. Matsuno $H$, Yudoh $K$, Katayama $R$, et al. The role of TNF-alpha in the pathogenesis of inflammation and joint destruction in rheumatoid arthritis (RA): a study using a human RA/SCID mouse chimera. Rheumatology (Oxford) 2002;41:329-337.

18. Mease PJ. Tumour necrosis factor (TNF) in psoriatic arthritis: pathophysiology and treatment with TNF inhibitors. Ann Rheum Dis 2002;61:298-304.

19. Sartor RB. Mechanisms of disease: pathogenesis of Crohn's disease and ulcerative colitis. Nat Clin Pract Gastroenterol Hepatol 2006;3:390-407.

20. Feldmann M, Maini RN. Anti-TNF alpha therapy of rheumatoid arthritis: what have we learned? Annu Rev Immunol 2001;19:163-196.

21. Kang EH, Lee YJ, Hyon JY, et al. Salivary cytokine profiles in primary Sjogren's syndrome differ from those in non-Sjogren sicca in terms of TNF-alpha levels and Th-1/Th-2 ratios. Clin Exp Rheumatol 2011;29: 970-976.

22. Fox Rl, Kang HI, Ando D, et al. Cytokine mRNA expression in salivary gland biopsies of Sjogren's syndrome. J Immunol 1994;152:5532-5539.

23. Howard Tripp N, Tarn J, Natasari A, et al. Fatigue in primary Sjogren's syndrome is associated with lower levels of proinflammatory cytokines. RMD Open 2016;2:e000282.

24. Maria NI, van Helden-Meeuwsen CG, Brkic Z, et al. Association of increased Treg cell levels with elevated indoleamine 2,3-dioxygenase activity and an imbalanced kynurenine pathway in interferon-positive primary Sjogren's syndrome. Arthritis Rheumatol 2016;68:1688-1699.

25. Kamachi M, Kawakami A, Yamasaki S, et al. Regulation of apoptotic cell death by cytokines in a human salivary gland cell line: distinct and synergistic mechanisms in apoptosis induced by tumor necrosis factor alpha and interferon gamma. J Lab Clin Med 2002;139:13-19.

26. Baker OJ, Camden JM, Redman RS, et al. Proinflammatory cytokines tumor necrosis factor-alpha and interferon-gamma alter tight junction structure and function in the rat parotid gland Par-C10 cell line. Am J Physiol Cell Physiol 2008;295:C1191-C1201.

27. Dorner T, Hucko M, Mayet WJ, et al. Enhanced membrane expression of the $52 \mathrm{kDa} \mathrm{Ro}(\mathrm{SS}-\mathrm{A})$ and $\mathrm{La}(\mathrm{SS}-\mathrm{B})$ antigens by human keratinocytes induced by TNF alpha. Ann Rheum Dis 1995;54:904-909.

28. Lai Z, Yin H, Cabrera-Perez J, et al. Aquaporin gene therapy corrects Sjogren's syndrome phenotype in mice. Proc Natl Acad Sci USA 2016;113:5694-5699.

29. Culp DJ, Quivey $\mathrm{RQ}$, Bowen $\mathrm{WH}$, et al. A mouse caries model and evaluation of aqp5-/- knockout mice. Caries Res 2005;39:448-454.

30. Ma T, Song $Y$, Gillespie A, et al. Defective secretion of saliva in transgenic mice lacking aquaporin-5 water channels. J Biol Chem 1999;274:20071-20074.

31. Yamamura $\mathrm{Y}$, Motegi $\mathrm{K}$, Kani $\mathrm{K}$, et al. TNF-alpha inhibits aquaporin 5 expression in human salivary gland acinar cells via suppression of histone H4 acetylation. J Cell Mol Med 2012;16:1766-1775.

32. Steinfeld SD, Demols P, Salmon I, et al. Infliximab in patients with primary Sjogren's syndrome: a pilot study. Arthritis Rheum 2001;44: 2371-2375.

33. Mariette $X$, Ravaud $P$, Steinfeld $S$, et al. Inefficacy of infliximab in primary Sjogren's syndrome: results of the randomized, controlled Trial of Remicade in Primary Sjogren's Syndrome (TRIPSS). Arthritis Rheum 2004;50:1270-1276.

34. Ewert $\mathrm{P}$, Aguilera $\mathrm{S}$, Alliende $\mathrm{C}$, et al. Disruption of tight junction structure in salivary glands from Sjogren's syndrome patients is linked to proinflammatory cytokine exposure. Arthritis Rheum 2010;62: 1280-1289. 
35. Sumida $T$, Tsuboi $H$, lizuka $M$, et al. The role of $M 3$ muscarinic acetylcholine receptor reactive $\mathrm{T}$ cells in Sjogren's syndrome: a critical review. J Autoimmun 2014;51:44-50.

36. Cha S, Singson E, Cornelius J, et al. Muscarinic acetylcholine type-3 receptor desensitization due to chronic exposure to Sjogren's syndrome-associated autoantibodies. J Rheumatol 2006;33: 296-306.

37. lizuka $M$, Wakamatsu $E$, Tsuboi $H$, et al. Pathogenic role of immune response to M3 muscarinic acetylcholine receptor in Sjogren's syndrome-like sialoadenitis. J Autoimmun 2010;35:383-389.

38. Aringer $M$, Smolen JS. The role of tumor necrosis factor-alpha in systemic lupus erythematosus. Arthritis Res Ther 2008;10:202.

39. Tornwald J, Fox H, Edwards $\mathrm{C}$, et al. Treatment with pegylated recombinant methionyl human soluble tumor necrosis factor-type 1 receptor prevents development of Sjögren's syndrome and diabetes in the NOD mouse model. Arthritis Rheum 1999;42:S403.

40. Vosters $\mathrm{JL}$, Yin $\mathrm{H}$, Roescher $\mathrm{N}$, et al. Local expression of tumor necrosis factor-receptor 1:immunoglobulin $G$ can induce salivary gland dysfunction in a murine model of Sjogren's syndrome. Arthritis Res Ther 2009;11:R189.

41. Choi K, Ni L, Jonakait GM. Fas ligation and tumor necrosis factor alpha activation of murine astrocytes promote heat shock factor-1 activation and heat shock protein expression leading to chemokine induction and cell survival. J Neurochem 2011;116:438-448.
42. Algood HM, Lin PL, Yankura D, et al. TNF influences chemokine expression of macrophages in vitro and that of CD11b+ cells in vivo during Mycobacterium tuberculosis infection. J Immunol 2004;172: 6846-6857.

43. Groom JR, Luster AD. CXCR3 ligands: redundant, collaborative and antagonistic functions. Immunol Cell Biol 2011;89:207-215.

44. Kramer JM, Klimatcheva E, Rothstein TL. CXCL13 is elevated in Sjogren's syndrome in mice and humans and is implicated in disease pathogenesis. J Leukoc Biol 2013;94:1079-1089.

45. Han BK, Kuzin I, Gaughan JP, et al. Baseline CXCL10 and CXCL13 levels are predictive biomarkers for tumor necrosis factor inhibitor therapy in patients with moderate to severe rheumatoid arthritis: a pilot, prospective study. Arthritis Res Ther 2015;18:93.

46. Baker OJ. Tight junctions in salivary epithelium. J Biomed Biotechnol 20102010; 278948.

47. Almoallim $\mathrm{H}, \mathrm{Al}-\mathrm{Ghamdi} \mathrm{Y}$, Almaghrabi $\mathrm{H}$, et al. Anti-tumor necrosis factor-alpha induced systemic lupus erythematosus. Open Rheumatol J 2012;6:315-319.

48. Aringer $M$, Steiner $G$, Graninger $W B$, et al. Effects of short-term infliximab therapy on autoantibodies in systemic lupus erythematosus. Arthritis Rheum 2007:56:274-279.

49. Aringer $M$, Graninger WB, Steiner $G$, et al. Safety and efficacy of tumor necrosis factor alpha blockade in systemic lupus erythematosus: an open-label study. Arthritis Rheum 2004;50:3161-3169. 